\title{
Year in review 2010: Critical Care - cardiac arrest and cardiopulmonary resuscitation
}

\author{
Jeffery C Metzger*, Alexander L Eastman and Paul E Pepe
}

\begin{abstract}
This review will summarize some of the data published in 2010 and focus on papers published in Critical Care in regard to cardiac arrest and cardiopulmonary resuscitation. In particular, we discuss the latest research in therapeutic hypothermia after cardiac arrest, including methods of inducing hypothermia, potential protective mechanisms, spontaneous hypothermia versus therapeutic hypothermia, and several predictors of outcome. Furthermore, we will discuss the effects of bystander-initiated cardiopulmonary resuscitation (CPR) in patients with physician-assisted advanced cardiac life support, the role of hypercapnea in near-death experiences during cardiac arrest, markers of endothelial injury and endothelial repair after CPR, and the prognostic value of cell-free plasma DNA as a marker of poor outcome after cardiac arrest.
\end{abstract}

\section{Introduction}

In 2010, a number of papers were published in the field of cardiac arrest and cardiopulmonary resuscitation (CPR). Critical Care provided us with some innovative and important data within these fields of research. This review will summarize some of the notable data published in 2010 and focus on papers published in Critical Care. For example, we discuss the latest research in therapeutic hypothermia after cardiac arrest and also review the effects of bystander-initiated cardiopulmonary resuscitation (BCPR), the role of hypercapnea in neardeath experiences (NDEs) during cardiac arrest, markers of endothelial injury after CPR, and the use of cell-free plasma DNA as a marker to predict outcome after CPR.

*Correspondence: jeffery.metzger@utsouthwestern.edu

Department of Surgery, University of Texas Southwestern Medical Center at Dallas, 5323 Harry Hines Boulevard, Mail Code 8579, Dallas, TX 75390-8579, USA

\section{Therapeutic hypothermia after cardiac arrest}

While the idea of therapeutic hypothermia is not new by any means (dating back to its recommended use by Hippocrates for wounded patients [1]), therapeutic hypothermia has been shown for almost a decade to decrease mortality and improve outcomes after cardiac arrest $[2,3]$. In 2010, we continued to learn about this life-saving therapeutic modality.

Several studies looked at the mechanisms of cooling patients. One study looked at the use of an external shower of water $\left(2^{\circ} \mathrm{C}\right)$ which achieved a median rate of cooling of $3^{\circ} \mathrm{C}$ per hour [4]. Another study showed that the Arctic Sun device (Medivance, Inc., Louisville, CO, USA) cooled, on average, 54 minutes faster than other external measures such as ice packets and blankets [5], whereas yet another study [6] compared endovascular cooling with external cooling and showed that endovascular cooling led to more time in the target temperature range, less temperature fluctuation, and more control during rewarming. It is currently recommended that cooling be achieved as soon as possible [6]. In a study in Critical Care, Škulec and colleagues [7] looked at the effectiveness of infusing 15 to $20 \mathrm{~mL} / \mathrm{kg}$ of $4^{\circ} \mathrm{C}$ saline intravenously in the pre-hospital environment and found an average decrease in the tympanic temperature of $1.4^{\circ} \mathrm{C}$ over the course of 42.8 minutes. They also found that the most effective cooling was achieved with a longer transport time and with a larger bolus of fluid administered.

While the current recommendation is rapid cooling, one study examined those patients admitted to an intensive care unit (ICU) after cardiac arrest with spontaneous hypothermia and investigated whether their outcomes were any different than those with therapeutically induced hypothermia since the former should have a decreased time to goal temperature. In their observational cohort study, den Hartog and colleagues [8] showed that patients with spontaneous hypothermia had a much higher likelihood of unfavorable outcome: odds ratio (OR) of 2.6, which increased to 3.8 after adjusting for age, presenting heart rhythm, and APACHE II (Acute Physiology and Chronic Health Evaluation II) and SOFA (Sequential Organ Failure Assessment) scores. Spontaneous hypothermia had already been shown to be a 
predictor of unfavorable outcome in trauma $[9,10]$ and sepsis $[11,12]$, but this was the first time that it had been shown in patients after cardiac arrest. While it was postulated that circulating cytokines had a role in hypothermia and unfavorable outcomes, Marik and Zaloga [13] showed no significant difference in circulating cytokines in septic patients who were hypothermic versus those who were febrile, despite a much higher incidence of organ dysfunction and unfavorable outcomes in the hypothermic group. However, in an article in Critical Care in 2010, Meybohm and colleagues [14] showed that, in pigs that underwent cardiac arrest followed by return of spontaneous circulation (ROSC), there was a significant upregulation of inflammatory cytokines but that those undergoing therapeutic hypothermia had a significantly smaller increase in cerebral tissue cytokine mRNA expression (interleukin (IL)- $1 \beta$, IL-6, IL-10, tumor necrosis factor-alpha, and intracellular adhesion molecule-1) as well as a lower level of tissue IL-1 $\beta$ protein levels compared with a normothermic group. This suggests at least part of the mechanism by which therapeutic hypothermia protects neurologic function after cardiac arrest.

One question that is often asked is who benefits from therapeutic hypothermia after ROSC. Aguila and colleagues [15] showed that survivors tended to be younger and in ventricular fibrillation (VF) on presentation, used aspirin prior to the arrest, required less epinephrine during the arrest, were less likely to be taking beta-blockers or angiotensin-converting enzyme (ACE) inhibitors, had no coronary artery disease (CAD), had preserved renal function, and had an ROSC of less than 20 minutes. In another article in Critical Care in 2010, Kagawa and colleagues [16] compared the impact of the time interval from collapse to ROSC and the impact of therapeutic hypothermia for the different time intervals. While they showed the expected increased survival as the interval from collapse to ROSC decreased, they also showed that therapeutic hypothermia may be beneficial in patients with a cardiopulmonary arrest (CPA) ROSC of greater than 15 minutes, but there was no significant difference in patients with CPA ROSC of 15 minutes or less.

\section{Bystander-initiated cardiopulmonary resuscitation}

Debate about the effectiveness of physician participation in pre-hospital emergency care has persisted for several decades. In the Japanese model, standard emergency medical services (EMS) care is supervised by the Fire and Disaster Management Agency of Japan. Within this system, an ambulance crew generally consists of three people, one of whom is an emergency life-saving technician (ELST) who has been 'extensively' trained in prehospital EMS. These ELSTs provide procedures including the use of the semiautomatic defibrillator, the use of supraglottic airway devices, and the infusion of Ringer's lactate and epinephrine - that are limited in scope [17]. While the Japanese EMS system consists of this one tier, several regional centers have further developed their own physician-staffed EMS systems. At present, 218 emergency and critical care centers are found in the 47 Japanese prefectures. Of these centers, 86 have physician-staffed pre-hospital advanced cardiac life support (ACLS) systems [18]. In these physician-staffed units, physicians carry out a wide variety of emergency treatments - including endotracheal intubation, the insertion of central venous catheters, and the infusion of many different medications, including catecholamines, lidocaine, anesthetic drugs, and even thrombolytics that exceed ELST capabilities.

In this prospective, population-based observational study, a group from the University of Tokyo sought to elucidate the effects of a combination of BCPR and the provision of ACLS by physician-staffed ambulances (PSAs), rather than by the aforementioned ELSTs (as is more commonly done), on survival with good cerebral outcome after out-of-hospital cardiac arrest (OHCA). Using a registry of all OHCA patients in Japan, the authors prospectively culled more than 95,000 patients whose cardiac arrests were witnessed by bystanders. These patients were then divided into four groups: group A (ACLS by ELSTs without BCPR), group B (ACLS by ELSTs with BCPR), group C (ACLS by physicians without $\mathrm{BCPR}$ ), and group $\mathrm{D}$ (ACLS by physicians with $\mathrm{BCPR}$ ). The Glasgow-Pittsburgh Cerebral Performance Category scoring system was used to evaluate neurologic outcomes.

According to a review of 95,072 bystander-witnessed arrests, $7,722(8.1 \%)$ were alive at 1 month, including 2,754 (2.9\%) with good cerebral outcome and 3,171 (3.3\%) with vegetative status or worse at 1 month. When group A was used as the control, the rates of good performance survival were significantly higher in group B (OR 2.23, 95\% confidence interval (CI) 2.05 to 2.42, $P<0.01$ ) and group D (OR 2.80, 95\% CI 2.28 to 3.43 , $P<0.01)$ and no significant difference was seen in group C (OR 1.18, 95\% CI 0.86 to $1.61, P=0.32$ ). The highest risk for the occurrence of a vegetative state at 1 month was seen in group C (OR 1.92, 95\% CI 1.55 to 2.37, $P<0.01)$. While it is clear from this study that the group with BCPR and physician-staffed ACLS (group D) had the best outcomes, it is also clear that physician involvement without accompanying BCPR (group C) can lead to an increase in the dreaded outcome of survival with neurologic devastation. This finding was so striking that the authors noted that more than $50 \%$ of 1 -month survivors in group C (ACLS by physicians without BCPR) were found either to be vegetative or to have de facto brain death. 
Despite the positive findings from the above study [18], several others have revealed no effect on survival or overall outcomes when physicians are involved in the pre-hospital care of OHCA. The most notable of these was a 5-year, prospective study from Oslo, Norway [19]. In this study, 1,128 OHCAs were managed by either PSAs or non-PSAs. In 977 cases, while the PSAs had better CPR quality as determined by shorter handoff intervals and pre-shock pauses and a higher proportion of patients who underwent endotracheal intubation, there were no differences between the groups with regard to short-term or long-term survival: $34 \%$ versus $33 \%$ $(P=0.74)$ achieved ROSC, $28 \%$ versus $25 \%(P=0.50)$ reached ICU admission, and 13\% versus 11\% $(P=0.28)$ were discharged home.

There is, ultimately, no conclusion that can be drawn when assessing the effects of physician involvement in pre-hospital ALCS for OHCA. Certainly, in many EMS systems, the costs and practicality of using physicians in the pre-hospital environment versus any derived benefit remain at question.

\section{Cell-free plasma DNA}

With regard to predicting outcomes after CPR, many factors, including serum lactate [20] and beta natriuretic peptide [21], have been examined, although none has proven to be entirely clinically useful. Cell-free plasma DNA, a more recently studied marker, has been found in increased concentrations in patients with either sepsis or septic shock [22]. In this paper, the authors from the Hospital Universitario La Paz in Madrid, Spain, sought to investigate whether a plasma DNA measurement in OHCA patients on arrival would be useful in predicting outcome [23].

During a 30-month study period, 113 consecutive adult patients arriving at the emergency department after nontraumatic, normothermic OHCA were recruited into the study. Ultimately, 85 patients met inclusion criteria for the study. Forty-eight (56.5\%) had acute myocardial infarction as their final diagnosis. The median cell-free plasma DNA concentration at admission was significantly higher in non-survivors at 24 hours than in survivors (5,520 genomic equivalents $(\mathrm{GE}) / \mathrm{mL}$ versus $2,810 \mathrm{GE} / \mathrm{mL}$, $P<0.01$ ), and the plasma DNA level at admission was significantly correlated with the total downtime $(r=0.579, P<0.001)$, maximum lactate concentration $(r=0.602, P<0.001)$, and the first 24-hour APACHE II score $(r=0.415, P<0.003)$. Additionally, the authors used receiver operating characteristic (ROC) curves to examine the use of plasma DNA as a predictor of 24-hour and in-hospital mortality. The area under the ROC curves for plasma DNA to predict 24-hour and in-hospital mortality were 0.796 (95\% CI 0.701 to 0.890$)$ and 0.652 ( $95 \%$ CI 0.533 to 0.770 ), respectively. When the same analysis was used, the best cutoff value of plasma DNA at admission for 24 -hour mortality was $4,340 \mathrm{GE} / \mathrm{mL}$ with a sensitivity of $76 \%$ and a specificity of $83 \%$. The authors concluded from these data that the measurement of plasma DNA on arrival in patients with OHCA might have some predictive value and provide some clinical utility.

Cell-free plasma DNA has been studied as a predictive index in other disease processes. In a precedent study, plasma DNA, when measured on admission to the ICU, proved to be an independent predictor of ICU mortality [24]. In this analysis of 255 patients from the Finnsepsis Study Group, one notable finding was that the predictive levels of plasma DNA were much higher than in the reviewed study by Arnalich and colleagues [23] (median plasma DNA level in non-survivors was $15,904 \mathrm{GE} / \mathrm{mL}$ ). It seems difficult, on the basis of the available data, to draw any truly useful conclusions from the measurement of plasma DNA in this setting.

\section{Near-death experiences and carbon dioxide}

Occurring in $11 \%$ to $23 \%$ of survivors of cardiac arrest, the NDE is described in the literature as a deep psychological experience with feelings of transcendence or a mythical encounter' [25] that typically occurs in a person close to death. While several theories have been proposed to explain these NDEs (including psychological, physical, and transcendental theories), none has been able to provide a clear and concise explanation for the phenomena [25]. Some physiologic theories have attempted to describe the NDE as part of the physiologic cascade of events surrounding death, although, again, none of these studies has proven to be definitive [26]. In this next paper, the authors examined the effect of the serum partial pressure of carbon dioxide $\left(\mathrm{pCO}_{2}\right)$ on the occurrence of NDEs in survivors of OHCA.

During an 18-month study period, Klemenc-Ketis and colleagues [27] studied survivors from OHCA in the ICUs of the three largest hospitals in Slovenia. To evaluate NDEs, investigators had study participants selfreport about the event on a 16-question evaluation tool that assessed the cognitive, affective, paranormal, and transcendental components of the experience. Scores of 7 or greater were designated the NDE group. With regard to data collection of the physiologic variables, data were obtained from patient charts from the beginning of resuscitative efforts until ROSC.

A total of 52 patients, of whom $21.2 \%(n=11)$ reported having an NDE according to the above criteria, were evaluated. Patients with a higher petCO $\mathrm{C}_{2}$ (partial pressure of end-tidal carbon dioxide) had more NDEs and the NDE score positively correlated with $\mathrm{pCO}_{2}(r=0.366$, $P=0.017)$. In multivariate analysis, elevated $\mathrm{pCO}_{2}$ was an independent predictor of NDE and a higher NDE score 
was associated with a higher $\mathrm{pCO}_{2}$ as well as higher levels of serum potassium and previous NDEs.

While several etiologic and even descriptive factors around the NDE remain nebulous, they appear to provide some information that is clinically useful. In another study, 72 patients who, in the 1980s, completed an NDE scale evaluation similar to the one described above were reevaluated more than 20 years later [28]. Interestingly, the participants' mean scores on the NDE scale did not change significantly, and the correlation between small score changes and time was not significant. These events clearly leave an indelible mark on those who perceive them and hence are an area that deserves further study. This mark has not just psychological implications but also, potentially, some precautionary ones as well. In a comprehensive study of 344 patients with ROSC after cardiac arrest in Dutch hospitals, investigators looked at many aspects of the $18 \%(n=62)$ of patients who reported having an NDE [29]. While many facets of the NDE are examined in this study, what is most striking is that significantly more patients who had an NDE died within 30 days of CPR $(P<0.0001)$.

\section{Endothelial damage after cardiopulmonary resuscitation}

In patients who undergo CPR and who have ROSC, the subsequent post-resuscitation period has proven to provide a complex constellation of critical care challenges. Some authors have attempted to describe this postresuscitation 'syndrome' as a whole-body ischemiareperfusion syndrome [30], whereas other have been more specific, attributing many aspects of this syndrome to endothelial activation following cardiac arrest itself [31]. This article, by Fink and colleagues [32] from the University Hospital of Freiburg, Germany, seeks to further characterize this endothelial activation.

Fink and colleagues examined 40 patients after CPR. These patients were then compared with a group of 30 patients with stable CAD and 9 healthy 'control' subjects. Direct endothelial damage was measured by examining two types of surrogates released after the endothelium is disrupted. The first of these, circulating endothelial cells (CECs), which have been described in a number of other disease states, including cardiovascular disease [33], seem to correlate with other markers of endothelial dysfunction. The second, endothelial microparticles, which have been found to have both procoagulant and inflammatory modulating properties [34], have also been found to be elevated in patients with cardiovascular disease. In order to evaluate the repair phase, the authors chose to evaluate circulating endothelial progenitor cells (EPCs) that have been shown to play a role in re-endothelialization and neovascularization [35].
There was a tremendous rise in the CEC count of patients who underwent resuscitation as compared with those with uneventful CAD $(4,494 \pm 1,294$ versus $312.7 \pm 41$ cells $/ \mathrm{mL}, P<0.001)$ or the healthy patient group ( $47.5 \pm 3.7$ cells $/ \mathrm{mL}, P<0.0005)$. Additionally, there was a positive correlation of CEC count with the duration of CPR, indicating continuing mechanical endothelial damage with the continuing trauma of resuscitation. The authors confirmed evidence of recovery and endothelial repair within 24 hours of ROSC as EPCs were significantly elevated in the post-CPR group compared with the stable CAD group $(1.16 \% \pm 0.41 \%$ versus $0.02 \% \pm 0.01 \%$ of lymphocytes, $P<0.005)$. The authors subsequently concluded that these results constitute evidence to describe a severe endothelial insult and subsequent repair that occur after ROSC. It has long been known that prolonged periods of resuscitation are associated with less-than-optimal outcomes after CPR and ROSC. With this paper, the authors have begun to try to quantify this effect by using the measurement of the above molecules.

\section{Conclusions}

During the past few years, many advances have been made in the fields of cardiac arrest and CPR. In research published in 2010 in Critical Care, we learned that the infusion of 15 to $20 \mathrm{~mL} / \mathrm{kg}$ of $4^{\circ} \mathrm{C}$ saline intravenously in the pre-hospital environment can significantly induce hypothermia as well as a potential mechanism for the improved outcomes in those with therapeutic hypothermia. We also learned more about who actually benefits from therapeutic hypothermia, namely those who were younger, were in VF on presentation, used aspirin prior to the arrest, required less epinephrine during the arrest, were less likely to be taking betablockers or ACE inhibitors, had no CAD, had preserved renal function, and had an ROSC of less than 20 minutes. Evidence suggests that patients presenting with spontaneous hypothermia after cardiac arrest tend not to do as well as those presenting with a normal body temperature. We learned that, although BCPR improved outcomes in OHCA, physician involvement in the prehospital arena led to a significant increase in the number of survivors remaining in a vegetative state. We also learned that cell-free plasma DNA is loosely predictive of poor outcomes after cardiac arrest, although its relatively low sensitivity and specificity likely preclude its use clinically. We saw an association between elevated $\mathrm{pCO}_{2}$ during resuscitation and the incidence of NDEs. Lastly, research showed that a significant endothelial insult occurs during cardiac arrest, and this may help explain some of the physiologic changes in the post-arrest period.

\section{Abbreviations}

$A C E$, angiotensin-converting enzyme; ACLS, advanced cardiac life support; APACHE II, Acute Physiology and Chronic Health Evaluation II; BCPR, bystander- 
initiated cardiopulmonary resuscitation; CAD, coronary artery disease; CEC, circulating endothelial cell; $\mathrm{Cl}$, confidence interval; $\mathrm{CPA}$, cardiopulmonary arrest; CPR, cardiopulmonary resuscitation; ELST, emergency life-saving technician; EMS, emergency medical services; EPC, endothelial progenitor cell; $\mathrm{GE}$, genomic equivalents; ICU, intensive care unit; IL, interleukin; NDE, neardeath experience; OHCA, out-of-hospital cardiac arrest; $\mathrm{OR}$, odds ratio; $\mathrm{PCO}_{2}$, partial pressure of carbon dioxide; PSA, physician-staffed ambulance; ROC, receiver operating characteristic; ROSC, return of spontaneous circulation; VF, ventricular fibrillation.

\section{Competing interests}

The authors declare that they have no competing interests.

Published: 5 December 2011

\section{References}

1. Polderman K: Application of therapeutic hypothermia in the ICU: opportunities and pitfalls of a promising treatment modality. Part 1: Indications and evidence. Intensive Care Med 2004, 30:556-575.

2. Hypothermia after Cardiac Arrest Study Group: Mild therapeutic hypothermia to improve the neurologic outcome after cardiac arrest. NEngl J Med 2002, 346:549-556.

3. Bernard S, Gray T, Buist M, Jones B, Silvester W, Gutteridge G, Smith K: Treatment of comatose survivors of out-of-hospital cardiac arrest with induced hypothermia. N Engl J Med 2002, 346:557-563.

4. Howes D, Ohley W, Dorian P, Klock C, Freedman R, Schock R, Krizanac D, Holzer M: Rapid induction of therapeutic hypothermia using convectiveimmersion surface cooling: Safety, efficacy and outcomes. Resuscitation 2010, 81:388-392.

5. Heard KJ, Peberdy MA, Sayre MR, Sanders A, Geocadin RG, Dixon SR, Larabee TM, Hiller K, Fiorello A, Paradis NA, O'Neil BJ: A randomized controlled trial comparing the Arctic Sun to standard cooling for induction of hypothermia after cardiac arrest. Resuscitation 2010, 81:9-14.

6. Nolan J, Deakin C, Soar J, Bottiger B, Smith G, Council ER: European Resuscitation Council guidelines for resuscitation 2005. Section 4. Adult advanced life support. Resuscitation 2005, 67 (Suppl 1):S39-S86.

7. Škulec R, Truhlár A, Seblová J, Dostál P, Cerný V: Pre-hospital cooling of patients following cardiac arrest is effective using even low volumes of cold saline. Crit Care 2010, 14:R231.

8. den Hartog A, de Pont A-C, Robillard L, Binnekade J, Schultz M, Horn J: Spontaneous hypothermia on intensive care unit admission is a predictor of unfavorable neurological outcome in patients after resuscitation: an observational cohort study. Crit Care 2010, 14:R121.

9. Wang H, Callaway C, Peitzman A, Tisherman S: Admission hypothermia and outcome after major trauma. Crit Care Med 2005, 33:1296-1301.

10. Kheirbeck T, Kochanek A, Alam H: Hypothermia in bleeding trauma: a friend or a foe? Scand J Trauma Resusc Emerg Med 2009, 17:65.

11. Peres Bota D, Lopes Ferreira F, Melot C, Vincent J: Body temperature alterations in the critically ill. Intensive Care Med 2004, 30:811-816.

12. Remick D, Xioa H: Hypothermia and sepsis. Front Biosci 2006, 11:1006-1013.

13. Marik PE, Zaloga GP: Hypothermia and cytokines in septic shock. Norasept II Study Investigators. North American study of the safety and efficacy of murine monoclonal antibody to tumor necrosis factor for the treatment of septic shock. Intensive Care Med 2000, 26:716-721.

14. Meybohm P, Gruenewald M, Zacharowski K, Albrecht M, Lucius R, Fosel N, Hensler J, Zitta K, Bein B: Mild hypothermia alone or in combination with anesthetic post-conditioning reduces expression of inflammatory cytokines in the cerebral cortex of pigs after cardiopulmonary resuscitation. Crit Care 2010, 14:R21.

15. Aguila A, Funderburk M, Guler A, McNitt S, Hallinan W, Daubert JP, Delehanty $J M$, Aktas MK: Clinical predictors of survival in patients treated with therapeutic hypothermia following cardiac arrest. Resuscitation 2010, 81:1621-1626.

16. Kagawa E, Inoue I, Kawagoe T, Ishihara M, Shimatani Y, Kurisu S, Nakama Y, Dai K, Otani T, Ikenaga H, Morimoto Y, Ejiri K, Oda N: Who benefits most from mild therapeutic hypothermia in coronary intervention era? A retrospective and propensity-matched study. Crit Care 2010, 14:R155.
17. Tanigawa K, Tanaka K: Emergency medical service systems in Japan: past, present, and future. Resuscitation 2006, 69:365-370

18. Ohshige K, Shimazaki S, Hirasawa H, Nakamura M, Kin H, Fujii C, Okuchi K, Yamamoto Y, Akashi K, Takeda J, Hanyuda T, Tochikubo O: Evaluation of outof-hospital cardiopulmonary resuscitation with resuscitative drugs: a prospective comparative study in Japan. Resuscitation 2005, 66:53-61.

19. Olasveengen TM, Lund-Kordahl I, Steen PA, Sunde K: Out-of hospital advanced life support with or without a physician: effects on quality of CPR and outcome. Resuscitation 2009, 80:1248-1252.

20. Kliegel A, Losert H, Sterz F, Holzer M, Zeiner A, Havel C, Laggner AN: Serial lactate determinations for prediction of outcome after cardiac arrest. Medicine (Baltimore) 2004, 83:274-279.

21. Sodeck GH, Domanovits H, Sterz F, Schillinger M, Losert H, Havel C, Kliegel A, Vlcek M, Frossard M, Laggner AN: Can brain natriuretic peptide predict outcome after cardiac arrest? An observational study. Resuscitation 2007, 74:439-445.

22. Rhodes A, Wort SJ, Thomas H, Collinson P, Bennett ED: Plasma DNA concentration as a predictor of mortality and sepsis in critically ill patients. Crit Care 2006, 10:R60.

23. Arnalich F, Menendez M, Lagos V, Ciria E, Quesada A, Codoceo R, Vazquez J, Lopez-Collazo E, Montiel C: Prognostic value of cell-free plasma DNA in patients with cardiac arrest outside the hospital: an observational cohort study. Crit Care 2010, 14:R47.

24. Saukkonen K, Lakkisto P, Pettila V, Varpula M, Karlsson S, Ruokonen E, Pulkki K: Cell-free plasma DNA as a predictor of outcome in severe sepsis and septic shock. Clin Chem 2008, 54:1000-1007.

25. Parnia S, Spearpoint K, Fenwick PB: Near death experiences, cognitive function and psychological outcomes of surviving cardiac arrest. Resuscitation 2007, 74:215-221

26. Parnia $S$, Waller DG, Yeates R, Fenwick $P$ : A qualitative and quantitative study of the incidence, features and aetiology of near death experiences in cardiac arrest survivors. Resuscitation 2001, 48:149-156.

27. Klemenc-Ketis Z, Kersnik J, Grmec S: The effect of carbon dioxide on neardeath experiences in out-of-hospital cardiac arrest survivors: a prospective observational study. Critical Care 2010, 14:R56.

28. Greyson B: Consistency of near-death experience accounts over two decades: are reports embellished over time? Resuscitation 2007, 73:407-411.

29. van Lommel P, van Wees R, Meyers $V$, Elfferich I: Near-death experience in survivors of cardiac arrest: a prospective study in the Netherlands. Lancet 2001, 358:2039-2045.

30. Adrie C, Laurent I, Monchi M, Cariou A, Dhainaou JF, Spaulding C. Postresuscitation disease after cardiac arrest: a sepsis-like syndrome? Curr Opin Crit Care 2004, 10:208-212.

31. Geppert A, Zorn G, Karth GD, Haumer M, Gwechenberger M, Koller-Strametz J, Heinz G, Huber K, Siostrzonek P: Soluble selectins and the systemic inflammatory response syndrome after successful cardiopulmonary resuscitation. Crit Care Med 2000, 28:2360-2365

32. Fink K, Schwarz M, Feldbrugge L, Sunkomat J, Schwab T, Bourgeois N, Olschewski M, von zur Muhlen C, Bode C, Busch $\mathrm{H}-\mathrm{J}$ : Severe endothelial injury and subsequent repair in patients after successful cardiopulmonary resuscitation. Critical Care 2010, 14:R104.

33. Mutin M, Canavy I, Blann A, Bory M, Sampol J, Dignat-George F: Direct evidence of endothelial injury in acute myocardial infarction and unstable angina by demonstration of circulating endothelial cells. Blood 1999, 93:2951-2958

34. Diamant M, Tushuizen ME, Sturk A, Nieuwland R: Cellular microparticles: new players in the field of vascular disease? Eur J Clin Invest 2004, 34:392-401.

35. Urbich C, Dimmeler S: Endothelial progenitor cells: characterization and role in vascular biology. Circ Res 2004, 95:343-353.

\section{doi:10.1186/cc10540}

Cite this article as: Metzger JC, et al: Year in review 2010: Critical Care cardiac arrest and cardiopulmonary resuscitation. Critical Care 2011, 15:239. 